\title{
Notizen für eine komplexe Beschreibung von Grande sertão: veredas
}

Guimarães Rosas kanonischer Roman Grande sertão: veredas gehört zur brasilianischen Tradition. Autor und Werk schreiben sich in die Geschichte dieses Landes ein und doch verbirgt sich hinter dem Anschein des Traditionellen das Neue und Unerwartete, das neue Möglichkeiten in der Konservierung und Tradierung des Vergangenen eröffnet. Der vorliegende Aufsatz möchte diese Möglichkeiten ausloten und anhand ausgewählter Textpassagen diskutieren.

Ich beginne bei den Hauptpersonen. Riobaldos Leidenschaft für Diadorim wird direkt am Anfang der Erzählung dargestellt. Diadorim erscheint ihm als Mann. Aber wie homoerotisch ist Grande sertão: veredas? Mancher sieht in Riobaldos Entdeckung und fortschreitender Akzeptanz seiner Begierde nach Diadorim seine konfliktreiche Selbstentdeckung als Homosexueller. Aber ist dies tatsächlich der Fall? Er entdeckt nicht sein sexuelles Interesse an Männern generell, sondern nur an einem einzigen Mann. Und biologisch gesehen ist dieser Mann eine Frau, die Riobaldo gerade durch ihre stereotypisch femininen Züge attraktiv erscheint: ihre sanfte Haut, der zarte Geruch, ihre Eleganz - die Art und Weise, wie sie die Wäsche wäscht. An sich sind die Attribute von Maskulinität und Femininität konventionell: Die Verhaltensweisen, Züge und die Erscheinung von Diadorim sind weiblich; sein Mut ist männlich. Die Attribute bewahren traditionelle Modi von Gegensätzen zwischen den Gendern; was diesen Kontrast destabilisiert, ist der Gebrauch der Attribute: Der Mann kann weiblich sein, wenn er Angst verspürt, die Frau kann männlich sein, wenn sie Mut zeigt. «A» unterscheidet sich ontologisch weiterhin von «B», es ist aber nicht möglich, zu sagen, dass «A» ausschließlich in «C» vorkommt und «B» nur in «D», denn «A» und «B» können ana$\log$ in $« \mathrm{C} »$ und «D» vorkommen. Das ist, was wir in Riobaldo und Diadorim sehen - jedoch ausschließlich in Riobaldo und Diadorim.

Denn die Destabilisierung der Herrschaft der Attribute Maskulinität und Femininität ist genau bestimmt: Die übrigen Charaktere sind Männer und Frauen. Eine erstaunliche Thematisierung der Homoerotik also, die das Thema offensichtlich anspricht, seine Wirkung jedoch abschwächt. Wir befinden uns im Jahre 1956. Nur wenige Jahre zuvor war Mário Donato wegen der Erotik in Presença de Anita aus der katholischen Kirche exkommuniziert worden: Wie wäre eine Thematisierung der Homoerotik aufgenommen worden, in der ein Jagunço sich in einen Mann mit eindeutig männlichen Charakterzügen verliebt und auf diese Weise sein generelles sexuelles Interesse für Männer entdeckt hätte? Ich kann mir nur 
vorstellen, dass die Rezeption von Grande sertão: veredas ganz anders gewesen wäre: Seiner Kanonisierung durch die Fachkritiken hätte dies nicht im Wege gestanden, sein Erfolg beim offenen Publikum wäre jedoch schwieriger vorstellbar gewesen - und auf diesen Erfolg zielte Rosa ab. Es findet sich hier ein strategischer Zug: Er übermittelt dem Leser das psychologische Drama eines Mannes, für den Homosexualität tabu ist, der jedoch allmählich sein sexuelles Interesse für einen anderen Mann erkennt und es sich eventuell eingesteht; dass er dieses Thema dem Leser von 1956 nahebringt, darin findet sich großer Wagemut. Doch das implizite Risiko wird letztlich mit der Enthüllung über Diadorim abgeschwächt: Der Wagemut wird schließlich durch eine Aushandlung vermittelt, in der das polemische Thema eingeführt und dann zurückgezogen wird. Trotz des Rückziehers pulsiert es weiterhin.

Gegenüber Männern und Frauen herrscht noch immer ein anderes traditionelles Element vor. Die Liebschaften Riobaldos schwanken zwischen Leidenschaft, Erotik und der Pflicht und zwar zwischen Diadorim, Nhorirá und Otacília. Die Leidenschaft ist groß, unkontrollierbar, aber nicht alltagstauglich; die Erotik an sich verspricht keine Dauerhaftigkeit; am Ende wird er die Sicherheit und Beständigkeit der Ehe wählen. Otacília ist Penelope: Sie ist Zuhause, Schutzort, zu ihr kehrt der Krieger nach der entscheidenden Schlacht heim, und mit ihr bleibt er für immer zusammen. In seinen Liebesentscheidungen ist Riobaldos Weg keine «Durchreise» und auch keine Selbstfindung: Er ist pragmatisch, realistisch, egoistisch und traditionalistisch bis zu dem Punkt, an dem er sich von seiner Intuition leiten lässt. An einem bestimmten Punkt spürt er, ahnt er voraus, dass Otacília die angemessene Wahl für seine Niederlassung in der Welt ist. Die konventionellen Alternativen werden nicht destabilisiert, sie bleiben als Gegensätze bestehen: Die Wahl drängt sich auf zwischen intensiver und bewegter Leidenschaft, Beständigkeit und Sesshaftwerdung aus Pflichtgefühl. Riobaldos Wahl für die Beständigkeit ist eindeutig und geht dem Tod Diadorims voran.

Im Allgemeinen ist das Handlungsuniversum maskulin, die Frauen existieren am Rande: Diadorim verkleidet sich als Mann, um sich in diesem Handlungsuniversum zu integrieren. Deutet dies auf eine Hierarchisierung der Geschlechter hin? Ich glaube nicht, denn jenes Universum ist zwar maskulin, aber moralisch verwerflich. Da sie außerhalb der Handlung stehen, werden die Frauen politisch freigesprochen und erhalten eine unvorhergesehene Würde: Inmitten der Bewahrung konventioneller Geschlechterattribute tritt die Bewertung der Frau als moralischer Reserve hervor.

Gehen wir weiter zum Protagonisten. Das Wort «Liebe» erscheint $142 \mathrm{Mal}$ in Grande sertão: veredas; das Wort «Angst» 194 Mal. Riobaldo spürt Angst, die für ihn eine wichtige Instanz der Subjektivierung ist: Es handelt sich um eine Emotion, die überwunden werden muss, oder zumindest in der Selbstbehauptung des 
«Ichs» verleugnet. Diese Selbstwahrnehmung ist kulturalisiert: Die Bewusstheit der eigenen Angst stört ihn, erinnern wir uns aber an die Unterscheidung von António Damásio, Neurowissenschaftler, nach der eine Emotion nicht mehr ist als eine chemische Entladung im Gehirn, die eine bestimmte Verhaltensreaktion hervorruft; «Gefühl» ist der Name, den wir dieser Reaktion geben - das Gefühl ist folglich eine Interpretation der Emotion. Riobaldos Problem ist es nicht, Angst zu spüren, sondern in einem Umfeld, in dem Mut überbewertet wird, sich ängstlich zu wissen. Das Problem ist nicht direkt die Emotion an sich, sondern das Konzept, das sie in diesem Umfeld erhält und das Konzept des Selbst, mit dem sich der Ängstliche gezwungenermaßen konfrontieren muss: Die Angst entmännlicht Riobaldo.

Aber bevor wir zu diesem Punkt übergehen, erinnern wir uns daran, dass alles in Grande sertão: veredas aus dem Blickwinkel des Erzählers präsentiert wird, einschließlich der Hierarchien, die die persönlichen Beziehungen in der Handlung organisieren: Held und gewöhnliches Individuum, Führer und Anhänger, Subjekt und «bloßes Individuum» (unabhhängig davon, ob dieses Individuum bewertet wird oder nicht). In meiner Ausgabe von Aguilar verbringt Riobaldo 260 der 360 Seiten in der Position des Individuums, nicht des Subjekts. Seine volle Subjektivierung, die Befriedigung seines Wunsches nach Verwirklichung, wird vertagt. Was steht auf dem Spiel?

Seine Mutter stirbt und er lebt daraufhin mit Selorico Mendes zusammen. Sein Leben ist bequem, aber Joca Ramiros Vision, die Auswirkungen der Kraft jener Jagunços weckt einen bestimmten Wunsch in ihm: Es sind Seiten geladen mit Ehrfurcht, Bewunderung und Spannung, auf denen Riobaldo versucht, Sinn auf diese noch unbekannte Art von Mensch, zu produzieren. Diese neue Beziehung setzt seine Meinung über Selorico herab, den er fortan als anspruchslosen Grundbesitzer sieht. Riobaldo flieht.

Er wird an Zé Bebelo verwiesen und mit ihm siegt er im ersten Krieg, aber sein unschlüssiges Verhältnis zu Bebelos Gewalt gegen die Jagunços, die er bewunderte, oder vielleicht sein Misstrauen in Bezug auf seine politischen Ambitionen bringt Riobaldo dazu, erneut zu flüchten: Hier würde er sein Verlangen nicht befriedigen. Zufällig trifft er Reinaldo-Diadorim wieder und tritt in Joca Ramiros Bande ein: Es ist ausschließlich Diadorims Gesellschaft, die starke sexuelle Spannung, die ihn dazu bringt, die Ziellosigkeit zu überwinden, um einer kontinuierlichen Beschäftigung nachzugehen. Warum war er so lange Zeit ohne Richtung, wenn doch das Leben mit Selorico Mendes und Zé Bebelo so vielversprechend erschien? Warum erschienen ihm jene Versprechen sozialer Integration unbefriedigend?

Nach dem Kontakt mit den Jagunços ist Riobaldo nicht mehr derselbe: Die Erfahrung erweckt in ihm einen gewissen mimetischen Wunsch und seine Subjektivität formt sich durch seinen Wunsch, das zu sein und zu haben, was jener Andere ist 
und hat. Er wünscht sich das, was der Andere sich wünscht, vor dem er Angst hat, den er beneidet und den er als Beispiel nimmt. Bis zum Ende der Erzählung modeliert sich sein Selbst, indem er den Wunsch des Anderen wünscht und die Bewunderung des Anderen anhand der Offenbahrung dessen begehrt, was der Andere selbst begehrt: Er ersehnt Joca Ramiros Willen nach Führung, Hermogenes Verlangen nach Stärke, Bebelos Streben nach Wissen (und künftig sogar das Bestreben nach Strategie, Planung und Vorherrschaft des Seo Habão), und er will dafür bewundert werden, ein starker und weiser Führer zu sein. Riobaldo will das Bild von sich sein, das er für die anderen zu projizieren versucht und in diesem Sinne kann sein Übergang von der Ziellosigkeit zur Handlung als ein Übergang in einen Zustand beschrieben werden, in dem sein geringer Stolz als gebildetes, ambitioniertes und dennoch untergeordnetes Individuum Platz macht für einen Zustand, in dem sein Stolz befriedigt werden kann - durch Imitation.

Das heißt, selbst in dieser offenbaren Selbstbehauptung behauptet sich Riobaldo nicht vollständig. Selbst als er zu Urutu-Branco wird, geht ihm Zé Bebelo niemals aus dem Kopf; er hört niemals auf, darüber nachzudenken, was Bebelo von dem, was er tut, halten würde. $\mathrm{Zu}$ einem bestimmten Zeitpunkt verletzt die Unwissenheit eines Fazendeiros über seine Leistungen, den er zufällig trifft, seine Eitelkeit: Es ist, als handle er kontinuierlich vor einem imaginären Publikum. Für die Realisierung seines Wunsches reicht Selbstzufriedenheit nicht aus, sondern nur der Beweis der Welt; sein Sinn für Status wird durch das fremde Urteil determiniert. Wenn Diadorim ihm dermaßen attraktiv erscheint, geschieht dies ebenfalls deshalb, da sie diejenige Tugend hat, die er niemals erreichen würde: Selbstsicherheit.

Ein unsicherer Riobaldo also: Ein Leben unter mütterlichem Schutz und dem Komfort des Paten, das in der Ehe mit Otacília und dem endlosen Zweifel über seine Vergangenheit endet. Wenn Grande sertão: veredas eine Bildungsroman-Komponente aufweist, wenn Riobaldos Ich durch sein Erleben der Welt geformt wird und wenn dieses bereits geformte Ich mit Abstand die entscheidenden Momente in seiner Formierung zu würdigen weiß, dann hätte eine solche Entwicklung unter mütterlichem Schutz begonnen, um unter andauernden Zweifeln in der Sicherheit mit Otacília zu enden. Die Erfahrung, die ihn zur Unabhängigwerdung des Ichs geführt haben könnte, würde ihn zu einem weiteren bequemen Grundbesitzer unter dem (scheinbar notwendigen) weiblichen Schutz machen - es ist ein seltsames Subjekt, das sich niemals zu verwirklichen scheint. Einmal mehr wurde ein Thema, das die Person des brasilianischen Romans modernisiert, eingeführt und im Moment seiner praktischen Verwirklichung entfernt: Wenn in Clarice Lispectors Perto do coração selvagem (1943) und in Ciranda de pedra (1954) von Lygia Fagundes Telles die Entstehung des Protagonisten in einer Erzählung von der Entwicklung der Persönlichkeit durch die Auseinandersetzung «Ich-Welt» inszeniert wurde, im Akt 
«der reinen Unterwerfung unter die Selbstregulierung» des Subjekts, betrieben durch einen «Prozess der Inkorporation, durch den das Individuum als [emanzipiertes] [...] Geschöpf konstituiert und reguliert wird ${ }^{1}$, dann bleibt Riobaldo in seinen ewigen Zweifeln, seiner Resignation und seinem Schutzbedürfnis weit hinter dieser «Emanzipation` zurück.

Seine Unsicherheit könnte lediglich durch einen Pakt mit dem Teufel überwunden werden. Jedoch gibt es keinerlei Indizien im Text, die darauf hindeuten, dass ein solcher Pakt eingegangen wird, und außerdem ist Riobaldo nicht Hermogenes: Er unterscheidet sich deutlich von ihm. Wenn es einen Moment gibt, in dem Guimarães Rosa versucht, Empathie für den Erzähler zu konstruieren, dann, wenn er inmitten der Gewalt auf das unnötige Schlechte verzichtet, indem er die Grausamkeit zurückweist und Rechtfertigung für die Gewalt verlangt. Hier tut sich das Problem der Begierde auf: Er wird von der Kraft des Jagunço verführt, von der Gewalt in Verwunderung gesetzt, die dieser bis in wollüstiger Weise praktiziert, aber er ist kein Jagunço - er ist ein Gebildeter und für ihn besteht Gewalt nicht widerspruchslos fort. Selbst in seiner Hybris als Urutu-Branco, in seiner Einsamkeit an der Macht und in seinem messianischen Glauben an seine eigenen Kapazitäten stimmt er Gewalt nur ab einem gewissen Niveau der Rechtfertigung zu, denn in gewisser Art und Weise muss er sich gut glauben. Das heißt, er rettet sich vor dem eigenen und fremden Urteil durch Selbstrechtfertigung, eine rational konsistente Art der Selbsttäuschung.

Gehen wir weiter zum Aufbau der Erzählung. Es ist möglich, so viel über den Plot von Grande sertão: veredas zu sagen, weil die offen von Riobaldo thematisierte Unsicherheit, Ambiguität und das Mysterium nicht ausschließen, dass die Ereignisse klar identifiziert werden können. Das Mysterium ernährt sich von Fakten unter konventioneller Sequenzierung: Zuerst das Ereignis, im Anschluss die Interpretation; zunächst erscheint das Objekt des Zweifels, danach der Ausdruck des Zweifels. Der zu interpretierende Stoff wird stark objektiviert, was die Motivationen der Handelnden einschließt. Sobald die Interpretation scheitert, wird es still. Wenn Riobaldo mystisch erscheint, dann handelt es sich hierbei um einen Mystizismus nach Wittgenstein: das, worüber man nicht sprechen kann, ist die Stille. In Wahrheit jedoch gibt es darin keinerlei Mystizismus, denn das Objekt der Stille ist rational artikuliert: Man kommt nicht durch mystische Interpretation $\mathrm{zu}$ ihr, sondern über die Vernunft. Der Analyseprozess an sich ist nicht mysteriös und das Mysteriöse ist nicht notwendigerweise ein ontologisches Attribut des Objekts, sondern eine epistemologische Begrenzung des Erkenntnissubjekts. Die Stille

1 Joseph R. Slaughter: Human Rights, Inc. New York: Fordham University Press 2007, S. 10 (unsere Übersetzung, Anmerkung des Übersetzers). 
wird mit ewiger Unbestimmtheit assoziiert, und das erzeugt mit Sicherheit Unruhe. Doch das offenbar unerklärbare Objekt ist objektiv gesehen erkannt.

Diese mysteriösen Elemente befinden sich unter denen, die Psychologie und Erkenntnistheorie traditionell beschäftigen: Die Erklärung der Ursachen des menschlichen Verhaltens (da unsere «tiefen» mentalen Prozesse unsichtbar sind), und die Bedeutung des Zufälligen, der Fügung und des Glücks in den weltlichen Ereignissen. Für Riobaldo kann der Verstand des Anderen manchmal interpretiert werden, er kann jedoch auch das reine Unbekannte sein. Gleichzeitig zeigen seine Treffen mit Reinaldo-Diadorim die Macht des Zufalls in einem Lebensweg.

Doch der Erzähler sorgt sich darum, seine eigene Ignoranz von den angebotenen Erklärungen der traditionellen - fast immer religiösen - Überzeugungen zu trennen. Riobaldo ist Ökumene, er glaubt ein bisschen etwas von allen Religionen, die er kennt. Dies ist wichtig, um eine glaubhafte Figur zu schaffen - dazu muss sie in jenem sozialen Kontext notwendigerweise religiös oder zumindest spiritualistisch sein - und gleichzeitig die traditionellen Überzeugungen auf Distanz halten: in Grande sertão: veredas sind die Figuren abergläubisch.

Wer glaubt an den Teufel? Guimarães Rosa, sein Leser sowie der Erzähler des Werks glauben nicht an ihn, und auch nicht Riobaldo selbst, dessen Beziehung mit dem Teufel durch die Notwendigkeit markiert ist, auf seine eigene Neigung zu reagieren, an etwas zu glauben, was er selbst als Aberglauben abwertet. Es gibt keine Anzeichen dafür, dass der Pakt geschehen ist und Riobaldo weiß das, was durch folgenden Punkt bekräftigt wird: Die erkenntnistheoretische Zusammensetzung des Narrativs ist zwischen klar erkennbaren Fakten (die Handlung könnte als Miniserie adaptiert werden) und einer offensichtlichen, zweckmäßig artikulierten Thematisierung des Mysteriums geteilt, einer Debatte über das Mysterium, die die Intrige nicht mystifiziert.

Eines der markantesten Ergebnisse dieses Gleichgewichts ist die Darstellung des Raums. Vom ersten Abschnitt an wird der geographische Raum des Sertão als unförmig, einheitslos und ohne präzise Grenzziehungen beschrieben. Es ist ein vom Menschen erschaffener Raum, der sich über Minas, Bahia und Goiás ausdehnt, dessen Ausdehnung mit der Ausbreitung von Netzwerken zusammenfällt, die Konfliktbeziehungen, kommerziellem Austausch, Kommunikation, Kooperation und Konkurrenz erkennbare Formen geben. Die Ausbreitung des Sertão stimmt zum Beispiel mit der Ausbreitung der wirtschaftlichen Geflechte überein, die sich in statischem Rahmen auf nicht wettbewerbsfähige Viehzucht und Ackerbau stützen, in denen die symbiotische Beziehung des Cangaço mit den Obersten und dessen Einschüchterung der Bevölkerung durch Isolation und Armut erklärt wird.

Geographisch unpräzise gewinnt der Raum als Kultur an Einheit. Nichts, was aus der Ferne kommt - Moden, Sprachen, Bräuche, Nachrichten, Gewohnheiten, 
Denkweisen - übt einen großen Einfluss aus. Frauen sind sesshaft und Bewegung ist überwiegend männlich; die Frauen sind Verbindungspunkte, sie sind die Häuser und die Länder, durch die sich die Netzwerke ziehen, und um sie herum werden Entscheidungen getroffen - aber sie selbst stehen still.

Es gibt sesshafte Arbeiter in den Dörfern. Cowboys und fahrende Händler zirkulieren. Fazendeiros sind sesshaft; Soldaten jagen die Jagunços und sind in Bewegung. Die Landschaft wird unterbrochen von Unterkünften, Landgütern und Dörfern, größere Städte liegen jedoch in weiter Ferne. Die Leere ist allgegenwärtig, und doch gibt es immer jemanden in der Nähe. Viele Informationen zirkulieren in dieser Leere, in den Treffen von Menschen auf der Durchreise. Der Landbesitz verleiht dem Raum durch ein Geflecht aus kleinen und großen Grundstücken wirtschaftliche und soziale Form. Obwohl das Territorium durch erkennbare Handlungsmuster organisiert ist, ist dieser Sertão jedoch instabil. Lokales Ethos und Kultur scheinen solide, uralt, aber ein historischer Wandel ist im Gange: Abstammungen und Zugehörigkeiten, die auf traditionellen Loyalitäten basieren, koexistieren mit dem Verrat des traditionellen Ethos, der der politischen und wirtschaftlichen Modernisierung der Republik implizit ist.

Es gibt auch einen zweiten Sertão, dargeboten in der ästhetischen und naturalistischen Wertschätzung der natürlichen Landschaft. Während der erste Sertão menschlich und kulturalisiert ist, ist der andere humboldtianisch: Er zeigt sich im Eintauchen in die Natur. Es ist Riobaldos ekstatischer Naturalismus, in dem der Anreiz zur Namensgebung und enzyklopädischen Katalogisierung der Naturgegebenheiten mit Verzauberung vermischt ist. Es sind punktuelle Momente, von geringem quantitativem Auftreten und ohne jegliche diegetische Entwicklungen die Wertschätzung des natürlich Schönen geschieht in der Unterbrechung der Handlung, wie in der Sequenz, in der Riobaldo nach Zé Bebeldos Verurteilung für zwei Monate auf einer Farm bleibt: Es ist ein Moment der Idylle, des Aufschubs der Haupthandlung, in dem er und Diadorim wie Daphnis und Chloe ein Gefühl des miteinander Teilens in der Natur erleben.

Es gibt noch einen dritten Raum im Sertão, der sich im Krieg konstituiert. Es ist kein kulturalisierter Ort, denn er ist reine Natur. Er ist auch nicht schön, denn ihn charakterisieren Hitze, Schmerz, Leid und Mühsal. Er ist erhaben: Er ruft in der Wertschätzung des Gleichgewichts keine Ruhe hervor, er erlegt dem Erzähler eine Flut von Worten auf, die niemals der Intensität seiner Erfahrung gerecht werden - die Sprache und die Vernunft scheitern an ihrer Repräsentation.

Die drei Räume vermischen sich nicht. Es gibt verschiedene Momente in der Handlung für jeden einzelnen Raum, was verschiedenen Handlungsweisen und Geisteszuständen des Erzählers entspricht. Auf der Ebene der Erzählung sind sie vergleich- und erkennbar, was auf unterschiedliche und ergänzende Arten der Wertschätzung des Sertão hindeutet. Dies geschieht deshalb, weil die erkenntnis- 
theoretischen Spaltungen gut funktionieren und der Präsentation jeden Raumes eine andere Art der Objektivierung vorbehalten ist: Der kulturalisierte Sertão wird in den Gewohnheiten, Praktiken, Lebensentwürfen der Einwohner unterschieden; der humboldtianische Sertão wird in der visuellen Schönheit erkannt und von der Neugier und dem analytischen Geist des distanzierten Beobachters genährt; der erhabene Sertão wird physisch durch Empfindung, Durst, den Einfluss feindlicher Natur auf den sich bewegenden Körper ertragen. $\mathrm{Zu}$ jeder Repräsentation des Raumes gehört sein Ort, sein Moment, seine Art der Erfahrung und des Intellekts.

Diese Klarheit in den Unterschieden zwischen den Darstellungen des Sertão wiederholt sich jedoch nicht in der Darstellung der brasilianischen Modernisierung. Es herrscht klare Instabilität. Die Republik will den Frieden unter staatlichem Gewaltmonopol fördern; wenn die Soldaten den Cangaço verfolgen, so tun sie dies, um seine parallelen Machtnetze ein für alle Mal zu zerstören. Die Republik möchte unter der Zentralgewalt in Rio de Janeiro den Respekt für den Gesellschaftsvertrag und das Gesetz festigen. Aber die Gewalt der Jagunços wird, obwohl sie durch Eigeninteresse motiviert ist, vom Schwertadel mit seinem Ehrenkodex, seiner Brüderlichkeit und seinem Respekt unter der natürlichen Autorität der Führerschaft beherrscht. Die zwei Machtnetzwerke stoßen aufeinander. Joca Ramiro und Medeiro Vaz verkörpern die natürliche Autorität des Schwertadels, neu ist jedoch die Entstehung der charismatischen Macht Zé Bebelos, die durch sein Wissen, seine Argumentationskraft und seine Taten legitimiert ist - es handelt sich um keine natürliche Macht, sondern um eine Kraft, die durch Verdienste erobert und vom Demos bewilligt wurde, und deren Erhaltung vom Erhalt seiner Popularität bedingt ist. Deshalb sieht von allen Anführern in der Erzählung nur Zé Bebelo die lokale Bevölkerung als Volk.

Der Unterschied liegt also zwischen dem Naturrecht und einem auf Recht und Argumentation beruhenden Gerechtigkeitsbegriff: Neue politische Formen belasten die bisherige Organisation auf der Grundlage des Verhältnisses von Grundbesitz und Eigentum. Im Gegensatz zu der Unpersönlichkeit des Staates steht der Egalitarismus der Jagunços, die durch Wahl und nicht durch Pflicht in dieses Leben gekommen sind, wie bei den Streitkräften. Der Zusammenhalt der Gruppe wird nicht durch Disziplin, sondern durch Treue verhängt. Ein Jagunço zu sein bringt wirtschaftliche Vorteile und lizenziert die Ausübung von Gewalt, aber es ist auch eine berufliche Option, die vom Kriegerethos und seinem Lob an Mut und Tapferkeit regiert wird. Die Gruppe hat einen Ehrenkodex, der auf ungeschriebenen Gesetzen basiert, und in dieser Identitätsaufschrift spielt es keine Rolle, ob sie Mineiros oder Brasilianer sind. Für die Kultur des Sertão ist die Idee der Nation irrelevant.

Die Führerschaft setzt sich auf natürliche Weise durch. Der Führer ist erfahren, selbstbewusst, weise, schlau, intelligent - und fair, innerhalb und außerhalb 
der Bande. Joca Ramiro ist die Art von Anführer, der Autorität ausstrahlt. Aber vielleicht ist er der letzte der natürlichen Führer der Cangaços, dessen Veranlagung zur Führung organisch, ontologisch und nicht erlernt scheint. Schon Medeiro Vaz erscheint rationaler: Er wäre ein moralischer Führer, ein Reiniger, der sich der Verteidigung des Guten verschreibt. Zé Bebelo wäre ein modernisierender Führer, von persönlicher Berechnung geleitet: Er möchte am Ende des Krieges einen Hobbes'schen Vertrag abschließen, der die Macht dem Staat überträgt; pragmatisch würde er die Jagunços im letzten Krieg leiten, die Regierung zur Befriedung führen und zur Zuerkennung des Gewaltmonopols an den Staat. Er versteht, dass das Verlangen nach Rache für Joca Ramiros Tod befriedigt werden müsste, damit Frieden herrschte und er sich zum Abgeordneten wählen lassen könnte, eine Art Verlangen, die die Republik im Sertão angestiftet hat: Die Modernisierung führt zur Veränderung der Vorstellungen und der politischen Ambitionen; soweit der Staat sich als Territorium versteht, transformiert er die Selbstdefinition, den Sinn für Strategie und den Willen des politisch ambitionierten Akteurs.

In Hermógenes und Ricardãos Augen verrät Joca Ramiro das Gruppengesetz, als er Zé Bebelo befreit, woraus sie schließen, dass es einen Vernichtungskrieg mit Waffenstillstand und einem Friedensvertrag geben müsse. Nach Riobaldos Sieg erbt er jedoch Besitztümer von Selorico Mendes und heiratet Otacília, womit er sich im Leben eines Grundbesitzers niederlässt, wie so viele Ex-Jagunços: Auf gewisse Art und Weise wurde Zé Bebelos Traum erfüllt und der republikanische Frieden setzt sich im Sertão durch. Im Präsens der Erzählung ist das Territorium durch die Eisenbahn, die Regierung und die Polizei angegliedert. Das Wort Regierung wird mehr als einmal großgeschrieben. ${ }^{2}$ Die Verwaltung des Territoriums reduziert den kleinen Grundbesitzer auf die Sesshaftigkeit, indem er ihn als Empfänger der Handlungen der politischen Macht positioniert: Während die archaischen Zeiten durch verteilte politische Initiativen charakterisiert wurden, ist die Initiative in modernen Zeiten im Staat zentralisiert. Vorher stellte der Sertão eine autonome Gesamtheit dar, die, obwohl gewalttätig und brutal, intern geregelt war. In der Gegenwart ist sie eine befriedete Gesamtheit, die jedoch abhängig und peripher ist. Zunächst in sich selbst autonom wird sie später in der Nation zur Peripherie. Dennoch bleiben einige Muster erhalten: Das Ende des Cangaço markiert das Ende einer historischen Phase, der Besitz von Boden überdauert jedoch die Zeit. Der beschleunigte Rhythmus des Cangaço verschwindet, die Trägheit des

2 Im Originaltext weist Dolabela Chagas hier auf die im Portugiesischen nicht obligatorischen Großschreibung des Wortes Governo (dt. Regierung) in Grande sertão: veredas hin (Anm. d. Üb.). 
ländlichen Lebens dauert fort: Sie ist kompatibel mit der archaisierenden Modernisierung der Republik.

Archaisierende Modernisierung: Am Ende herrscht Frieden und der Jagunço wird Landbesitzer, aber das Archaische macht auf blutige Weise dem Neuen Platz - es ist ein archaischer Übergang zur Moderne. Die republikanische Pax zwingt Riobaldo zur Resignation: Der Staat hat gewonnen und seine Identität als Jagunço, die ihn zuvor definiert hatte, muss unterdrückt und versteckt werden. Riobaldo fügt sich: Er erlebt die Gewalt der Polizei, erkennt die Willkür des Staates und kann nichts dagegen tun. In dieser Lähmung wird er von der Erinnerung an die Intensität des Cangaço belagert, jener edlen, gewalttätigen und heroischen Welt, die ihm Erfahrungen bot, die das geregelte sesshafte Leben eines Fazendeiro niemals bieten würde. In der Gegenwart herrscht eine lineare und kontinuierliche Zeit. Es ist keine offene Welt, in der der persönliche Wert von Mut, Tapferkeit, Antrieb, Führung - wo Verdienst von persönlichem Wert diktiert wird - herrühren würde. Jetzt ist sein Ort durch den Besitz von Boden bestimmt und die Autorität der erworbenen Weisheit wird vom praktischem Tun nicht verlangt: Ist es das, wohin er gekommen ist? Ist das die ersehnte Modernisierung?

In Seo Habão hatte er die liberale Wirtschaft gefunden: Geld, Akkumulation, Planung. In Zé Bebelo hatte er eine liberale Politik gefunden: Repräsentation, Legalität, Staatsbürgerschaft, delegierte Macht. Die beiden Figuren lassen den Kriegerstatus zur Vergangenheit werden: In der Moderne herrscht das in Ricardão so kritisierte Eigeninteresse; zuletzt setzen sich Berufung und Bestimmung durch, inmitten einer heftigen und brutalen Gemeinschaft, die jedoch für die individuelle Leistung offen ist (wie in den alten Kriegen, jeder Jagunço hatte seinen Anteil an Ruhm und Beute). Wenn der Staat das Ablassen von der Eigeninitiative durchsetzt, wird der Sertão zur Peripherie und verliert seine Selbstorganisation: Trotz der Ungleichheit, Brutalität und Rohheit (im Endeffekt eine Synekdoche Brasiliens), war der Sertão selbstgesteuert und ausgestattet mit Unternehmergeist und jetzt ist er betäubt. Das ist die politische Ambiguität von Riobaldo: Gewalt war schlecht, Frieden ist schlecht. Die Regierung scheint ihm notwendig zu sein, aber sie hat ihn zu einer kleinen Position in der Welt verurteilt: Mit diesem Groll wird er für immer leben.

In Grande sertão: veredas zeigt sich dieser Blick auf die Geschichte Brasiliens in einem komplexen Verhältnis zur Geschichte der brasilianischen Literatur, die von einer Mischung aus Innovation und Versöhnung geprägt ist. Innovation und Versöhnung: Es ist eine innovative Sprache, detailliert und poetisiert, die jedoch eine mimetische Umgangssprache heraufbeschwört (die Mundart des Sertão); eine Sprache, die die Mundart des Sertão in der Vorstellung hervorruft, aber nur in Rosas Werk existiert: Niemand spricht so. Die Handlung hat eine innovative Struktur ohne Kapitelunterteilung, aber mit einer konventionellen Einteilung in 
Absätze: Jeder Absatz entwickelt ein einziges Thema und der Wechsel von einem Absatz zum nächsten zeigt den Übergang zu einem anderen Thema an, was auch im Anfangsabschnitt des Werkes geschieht, bevor die Handlung sich entfaltet. Die Erzählung hat eine lange Einführung, faulknerianisch in ihrer zeitlichen Struktur und anders als alles, was im brasilianischen Roman bisher realisiert wurde, was in der Folge einen herkömmlichen zeitlichen Aufbau in mindestens drei Aspekten ermöglicht.

Der erste ist der offensichtlichste: Nach dem ersten, schwer zu lesenden Abschnitt wird die Zeitachse linear und die faulknerianische Desorientierung bleibt zurück. Der zweite Aspekt ist die klare Trennung der Rhythmen des Abenteuers im Erzählkontinuum, der Idylle, des Lernens durch zwischenmenschliche Geselligkeit, des Erinnerns: für jede Art von Erfahrung ein eigener Rhythmus, konventionell abgegrenzt durch Spannung und plötzliche Beschleunigung des Abenteuers, durch Dauer und Beständigkeit der Idylle, durch die Schnelligkeit des Dialogs, der durch die angehaltene Zeit der Erinnerung zur Einsicht des Erlernten führt. Und das dritte konventionelle Element in der Zeitkonstruktion ist die aus dem Roman des neunzehnten Jahrhunderts geerbte Balance in der Erzählung zwischen Ereignissen und fillers: Zahlreiche Ereignisse geschehen (Riobaldos Leben wurde durcheinander gebracht), aber sie sind in schnellen Abschnitten konzentriert und passieren in einem Abstand von langen Abschnitten, in denen nichts passiert - diejenigen fillers, die Jane Austen nach Franco Morettis Lektüre verwendet hat, um dem alltäglichen Leben «Regelmäßigkeit» und «Stil» zu verleihen. ${ }^{3}$ Es sind Sequenzen, die Gewohnheiten, Praktiken und Routinen darstellen, wodurch die individuellen und das kollektive Leben in ihren Rhythmen charakterisiert werden, ihrem Werte- und Erwartungskodex, in ihren materiellen und institutionellen Fundamenten und in ihren Modi der Öffnung und Beschränkung der Möglichkeiten, die typisch für diese fiktive Welt sind. In Grande sertão: veredas demonstrieren die fillers die Routine der Fazenda und der Jagunços, ihre Arten des Zusammenlebens, Sprachen und Überzeugungen, im Gehorsam gegenüber der traditionellen Konstruktion der Zeit, in der die Ereignisse akzentuieren und die fillers die Erzählung strukturieren, in einer Figur-Hintergrund Beziehung zwischen dem punktuellen Ereignis und dem Fortlauf des täglichen Lebens.

Ein letztes versöhnliches Element von Grande sertão: veredas wird häufig kommentiert: die Kombination von Universalität, Altertümlichkeit oder Zeitlosigkeit verbunden mit verschiedenen Charakteren (Joca Ramiro als Agamemnon, Otacília als Penelope, Riobaldo als Faust...), mit regionalistischem Wind, der

3 Franco Moretti: The serious century. In: Moretti, Franco (Hg.). The Novel. Vol. 1: History, Geography, and Culture. Princeton: Princeton University Press 2006, S. 364-400. 
durch die soziologische Inschrift geweckt wird und in der fiktionalen Welt kulturalisiert wird. Man kann sagen, dass diese Gleichung zu jener Zeit kein Problem mehr darstellte: In einem herderschen Geist, der in der Spontanität des gemeinen Mannes die Manifestation einer universellen Wahrheit identifizierte, war schon im Prolog von La vuelta de Martín Fierro 1879 der Gaucho der Pampas dadurch gekennzeichnet:

Wie einzigartig es ist [...], unsere unkultiviertesten Bauern zu hören, die in zwei klaren und einfachen Versen die Maximen und moralischen Gedanken ausdrücken, die die alten Nationen als einen unschätzbaren Schatz ihrer sprichwörtlichen Weisheit behielten, die die Griechen mit Ehrfurcht vor dem Munde ihrer tiefsten Weisen hörten. [...] Es gibt eine gewisse intime Ähnlichkeit, eine gewisse mysteriöse Identität unter all jenen Rassen der Welt, die ausschließlich im großen Buch der Natur studieren. ${ }^{4}$

Die maximale Spontanität ergibt sich aus dem maximalen Eintauchen in das natürliche Kontinuum, und so offenbart sich Weisheit: So ist der Gaucho nicht nur Argentinier, sondern universell. Aber in Grande sertão: veredas ist diese Gleichung angespannt, denn die Universalität bestimmter Figuren entspringt ausschließlich Riobaldos Vision - sie sind so in den Augen seines mimetischen Verlangens -, während die regionalistische Komponente des Werkes direkt im ersten Absatz durch das Eindringen des Narratärs gebrochen wird, der in der Erzählung eine Stadt-Land-Mediation internalisiert, die im Roman der 30er Jahre nicht existierte: Der Ich-Erzähler wendet sich seiner kulturellen Alterität zu und weiß, dass er seine Region jemandem präsentieren muss, der sie niemals ganz verstehen wird. Das Werk fügt sich so in die Geschichte des brasilianischen Romans ein und schöpft seine Unterschiedlichkeit aus. Mit allen hier genannten Merkmalen tritt kein modernistischer Grande sertão: veredas auf, der mit einem einseitigen Sinn für Bruch oder Innovation als Selbstzweck versehen ist. In ihm besteht Kontinuität mit der Vergangenheitsliteratur und Aushandlung mit der gegenwärtigen Öffentlichkeit. Die Arbeit befasst sich sowohl mit dem Intellekt, der sich im Regionalismus der 30er Jahre formt, als auch mit der Kritik, die der Kanonisierung des Modernismus diente (indem sie neue Formalismen in der künstlerischen Theorie und Praxis förderte) und gleichzeitig das Erbe der Romantik unter der Mediation des Existenzialismus erneuerte.

Der Regionalismus wird in der Geographie des kulturalisierten Sertão heraufbeschwört, die Romantik in der humboldtschen Repräsentation der Naturlandschaft, der Existenzialismus in der Aufspaltung der Mysterien der persönlichen

4 José Hernández: Martín Fierro. Barcelona: Editorial Sol 2000, S. 91-92 (unsere Übersetzung, Anm. d. Üb.). 
Motivationen, der Modernismus in der faulknerianschen Konstruktion der Handlung und der Formalismus in der sprachlichen Innovation. Diese Mischung bestimmt den Ort des Werkes als historischen Attraktor: Sein Erbe ist diskret unter den Schriftstellern - wie viele brasilianische Romanautoren sind eindeutig rosianisch? Aber die Kritiker hörten nie auf, den Roman zu kommentieren. Grande sertão: veredas ist noch immer Nationalliteratur, aber es brachte eine Wende hervor: Das Werk nahm eindeutig einen Platz in der Geschichte des brasilianischen Romans ein und damit gewann die Nationalliteratur ein Denkmal - und verlor gleichzeitig an Kraft, denn es war ein Denkmal des brasilianischen Romans, der erfordert, nicht nur als Brasilianer gelesen zu werden. Grande sertão: veredas heute zu lesen, bedeutet mindestens vier Epochen der Literatur und des brasilianischen Literatursystems zu durchlaufen. Hier finden wir die sertões: veredas. Die dualistische Interpretation des Landes durch den Kontrast zwischen Stadt und Sertão ist dort wirksam. In dieser Hinsicht ist Guimarães Rosa ein Erbe von Euclides da Cunha. Aber Grande sertão: veredas lässt von der Vermittlung der Wissenschaften (Natur- sowie Sozialwissenschaften) ab, die in Euclides und im Regionalismus der 30er präsent waren, um eine Kulturalisierung der Region zu betreiben, die sie symbolisch aufwertet, was die Entstehung einer anthropologischen Sensibilität markiert, die bereit ist, die Kulturen in ihren eigenen Begiffen $\mathrm{zu}$ interpretieren, und so dazu tendiert, Hierarchien umzukehren, um im Anderen Elemente zu würdigen, die in der Ausgangskultur des Autors und seiner Leser abwesend sind. Es war eine Geste, die der von Ariano Suassuna in O Auto da Compadecida ${ }^{5}$ entsprach, eine Arbeit, die gleichzeitig die Landkultur einer anderen Region des Landes symbolisch aufwertete, um ihren Status in der nationalen Vorstellung zu verändern. In Grande sertão: veredas finden wir auch José Lins do Regos Fogo Morto, Jorge Amados Terras do sem fim sowie Cascalho von Herberto Sales: Nach der ewigen Gegenwart des Regionalismus der 30er - und seiner statischen Darstellung der sozialen und menschlichen Landschaft - kommt am Anfang der 40er Jahre der Moment der Historisierung der Republik als ein Moment der Institutionalisierung des Archaismus und der Gewalt durch den Staat, die in unserer früheren Geschichte abgelagert sind. Wie bei diesen Arbeiten ging es bei Rosas Handlung um die Expansion des Staates durch das Territorium mit den Archaismen, die dieser Modernisierungsprozess bewahren sollte. Daher die Zweideutigkeit der Befriedung: Der Sertão wird unter anhaltender Gewalt zur Peripherie, ohne das Versprechen nach Gerechtigkeit.

In Grande sertão: veredas finden wir den Anfang der 50er Jahre wieder, als die brasilianische Literaturszene geteilt wurde vom Existentialismus und den Litera-

5 Titel in der deutschen Übersetzung von Willy Keller: Das Testament des Hundes oder das Spiel von Unserer Lieben Frau der Mitleidvollen (Anm. d. Üb.). 
turen des Ichs von Adalgisa Nery, Gustavo Corção, Lygia Fagundes Telles, Carlos Heitor Cony und Clarice Lispector, vom Regionalismus von Bernardo Élis und Mário Palmério (die ebenfalls der Erzählung der Ausweitung des Staates im Territorium gewidmet sind) und von der formalen Innovation des Konkretismus, die sich alle an ein heterogenes Publikum richten, welches vom Kanon des 19. Jahrhunderts geschult wurde und welches auch den Modernismo von 1922 und den Roman der 30er kanonisierte. Im Dialog mit einem vielfältigen synchronischen Bereich, der die gleichzeitige Akzeptanz von Lesern verschiedener Geschmäcker und Voraussetzungen sucht, verdichtet die Arbeit von Rosa nicht nur die Geschichte des brasilianischen Romans, sondern auch die Gegenwart - er verwickelt in einer einzigartigen Leistung Elemente verschiedener Literaturen (der Vergangenheit und der Gegenwart), die gleichzeitig von der Öffentlichkeit geschätzt und in Gelehrtenkreisen legitimiert sind.

Schließlich ist es unmöglich, das explodierende akademische Interesse an der Arbeit bei der Lektüre nicht wiederzuentdecken. Es ist unmöglich, nicht zu sehen, dass Grande sertão: veredas jahrzehntelange Diskussionen in den Universitäten hervorgebracht hat, und meine Diskussion könnte erklären, wie das Werk in seiner Komplexität solch einen vielfältigen Intellekt befriedigen und dieses Interesse im Laufe der Zeit erneuern konnte. Es fiel in die Gunst der Soziologen der USP und der Formalisten der PUC von São Paulo: Antonio Candido und Haroldo de Campos. Es wurde von Heideggerianern, Existenzialisten und Psychoanalytikern bewundert. Es wurde leidenschaftlich von Gender-Aktivisten, der Ökokritik und der Dekonstruktion diskutiert. Es wird von Germanisten, Hellenisten und Lateinamerikanisten studiert. Und alle identifizieren in ihm etwas, was sie im Speziellen interessiert: Es gibt niemanden, der in Grande sertão: veredas nicht ein Modell, Beispiel oder einen Ausdruck von etwas sieht, das direkt auf seine Disziplin oder kritische Perspektive bezogen werden kann - auch wenn dies häufig zu einer selektiven Lektüre oder Verzerrung des Textes in Richtung eines spezifischen Interesses führt. In jedem Fall handelt es sich um einen Text, dessen Komplexität diese Vielfalt an Aneignungen ermöglicht oder gar fördert.

Das dem Werk eigene bestimmt somit seine fortdauernde Präsenz in der Zeit mit. Die Konstruktion von Grande sertão: veredas setzte die Kontinuität und Bedeutung der Tradition des Romans der 30er und seines euklidischen Standards der nationalen Interpretation voraus und positionierte sich neben Literaturen, die neue Traditionen und Linien im brasilianischen Roman eröffneten. Auch wenn die Zeitgenossen es nicht so gelesen haben, gab es im Rückblick eine zeitliche Wende, in der Elemente, die bald in der Vergangenheit liegen sollten, immer noch das Proszenium besetzten, während Leitlinien der nahen Zukunft Präsenz und Legitimation gewannen. Was wäre das wohl für eine Zukunft? 